\title{
Article \\ Electrically Tunable Terahertz Focusing Modulator Enabled by Liquid Crystal Integrated Dielectric Metasurface
}

\author{
Yanchun Shen ${ }^{1,2}$, Zhixiong Shen ${ }^{3}$, Yuye Wang ${ }^{2, *}$, Degang $\mathrm{Xu}^{2, *}$ and Wei $\mathrm{Hu}^{3}$ (D) \\ 1 Information Engineering Institute, Guangzhou Railway Polytechnic, Guangzhou 510432, China; \\ shenyanchun@gtxy.edu.cn \\ 2 Institute of Laser and Optoelectronics, School of Precision Instruments and Optoelectronics Engineering, \\ Tianjin University, Tianjin 300072, China \\ 3 Key Laboratory of Intelligent Optical Sensing and Manipulation, College of Engineering and Applied \\ Sciences, Nanjing University, Nanjing 210093, China; dz1634004@smail.nju.edu.cn (Z.S.); \\ huwei@nju.edu.cn (W.H.) \\ * Correspondence: yuyewang@tju.edu.cn (Y.W.); xudegang@tju.edu.cn (D.X.)
}

check for updates

Citation: Shen, Y.; Shen, Z.; Wang, Y.; $\mathrm{Xu}, \mathrm{D}$;; Hu, W. Electrically Tunable Terahertz Focusing Modulator Enabled by Liquid Crystal Integrated Dielectric Metasurface. Crystals 2021, 11, 514. https://doi.org/10.3390/ cryst11050514

Academic Editors: Maxim

V. Gorkunov and Anatoliy

V. Glushchenko

Received: 14 April 2021

Accepted: 29 April 2021

Published: 6 May 2021

Publisher's Note: MDPI stays neutral with regard to jurisdictional claims in published maps and institutional affiliations.

Copyright: (c) 2021 by the authors. Licensee MDPI, Basel, Switzerland. This article is an open access article distributed under the terms and conditions of the Creative Commons Attribution (CC BY) license (https:// creativecommons.org/licenses/by/ $4.0 /)$

\begin{abstract}
Active lenses with focal tunable properties are highly desired in the modern imaging systems from the visible to the microwaves. In this paper, we demonstrate a terahertz $(\mathrm{THz})$ lens with electrically switchable focal length. It is composed of a large-birefringence liquid crystal (LC) layer infiltrating a dielectric metasurface. When the birefringence of LC is tuned with an external bias, the phase shift of a single meta-unit will change. With parameter sweep using the finite-different time-domain (FDTD) simulation method, meta-units with varying geometries are optimized to achieve a focal length switchable metalens. The numerical results show that the focal length can be switched between $8.3 \mathrm{~mm}$ and $10.5 \mathrm{~mm}$ at bias OFF and ON states, respectively, which is consistent with the design. A feasible fabrication procedure of the lens is further discussed. Such a device can be designed beyond the $\mathrm{THz}$ band to the visible or the microwaves, and may be widely applied in integrated imaging systems.
\end{abstract}

Keywords: dielectric metasurface; liquid crystal; focal tunable lens; terahertz

\section{Introduction}

The lens is an important and indispensable component in the imaging system. The traditional lens can be classified into the refractive optical elements (ROEs), which are made of polished curved crystals or polymers and rely on the phase accumulation difference along the propagation of light [1]. The disadvantages are that they are heavy and lack multi-functions. With the development of imaging systems, more requirements are proposed for the advance of lenses, such as integrability, broadband operation, achromatism and tunability. The recently developed planar metasurface provides a solution to fabricate high-performance metasurface lenses; namely, metalenses [2-5]. A metasurface is composed of an artificial electromagnetic unit structure in the two-dimensional scale [6]. The wavefront manipulation can be accomplished in a subwavelength resolution to achieve multi-functional metalenses. Until now, high efficiency all-dielectric metalenses [7], spindecoupled focusing [8] and broadband achromatic metalenses [9-12] have been realized, covering a broadband from the visible to the microwaves. Although the metasurface has been integrated with active materials to realize tunable functions [13], a metalens with tunable focal length still remains a challenge.

Liquid crystal (LC) is a birefringent material with excellent responsivity to the external light, temperature and electromagnetic fields. Apart from the wide applications in the display industry, it also plays a vital role in the non-display area. LC-based spatial light modulators (SLMs) are widely used in optical beam shaping [14]. LC geometric phase elements are capable of special beam generation, e.g., vortex beam, vector beam or Airy 
beam [15-17]. Some specific LCs, such as the dual-frequency LC and ferroelectric LC, can respond quickly to alternating currents, which can be used in optical switching and filtering $[18,19]$. Recently, LCs have also been integrated with metasurfaces to endow these devices tunability [20-24]. In this way, active filters and dynamic meta-absorbers are realized. However, these devices mainly have uniform meta-unit arrays, limiting their applications in filtering. The further exploitation of LC-integrated metasurfaces to dynamically control the wavefront is urgent, e.g., tunable focusing.

In this paper, we integrate a large-birefringence LC with a dielectric metasurface to demonstrate a metalens with an electrically switchable focal length in the terahertz (THz) band. The metasurface is composed of rectangular silicon pillars with varying length and width from the lens center to edge, forming a lens phase profile. The phase shift of the meta-unit will change due to the birefringence variation of LC with an external bias, resulting in an electrically switchable focal length. With the numerical simulation, it shows that the focal length can be switched between $8.3 \mathrm{~mm}$ and $10.5 \mathrm{~mm}$ at bias OFF and ON states, respectively. A feasible fabrication procedure is further discussed. Such a metalens may be widely used in imaging systems from the visible to the microwaves.

\section{Principle and Design}

As shown in Figure 1a, the metalens is composed of the upper substrate, the lower dielectric metasurface and the middle LC layer. Both alignment layers covered on the substrate and metasurface provide a homogeneous pre-alignment for LCs along the $x$ axis. The substrate and metasurface should be conductive to electrically switch the LC orientation. In the $\mathrm{THz}$ band, silicon can be chosen for its high $\mathrm{THz}$ transmittance and conductivity [25]. When a saturated bias is applied on both the substrate and metasurface, LCs will reorient along the $z$-axis (Figure $1 \mathrm{~b}$ ). In this way, the phase modulation of the transmitted $\mathrm{THz}$ wave will change, resulting in a varied focal length. A rectangular silicon pillar meta-unit covered with LC is shown in Figure 1c with its dimension parameters. When illuminated with the $x$-polarized $\mathrm{THz}$ wave, each pillar can be regarded as an effective waveguide [26]. The phase of the transmitted wave can be given by $n_{\mathrm{eff}} \cdot h \cdot 2 \pi / \lambda$, where $n_{\text {eff }}, h$ and $\lambda$ are the effective refractive index, the pillar height and the incident wavelength, respectively. Thus, the simulated phase of the transmitted $x$-polarized $\mathrm{THz}$ wave exhibits a linear dispersion, as shown in Figure 1d. When the external bias turns from OFF to ON, the orientation of LC changes from $x$-axis to $z$-axis, contributing to a distinct phase shift (Figure 1d). This can be attributed to two factors. Firstly, the height $H$ of LC is comparable to the THz wavelength. The refractive index change of LC from $n_{\mathrm{e}}$ to $n_{\mathrm{o}}$ will induce a propagation phase variation. Secondly, the resonant property of the silicon pillar is sensitive to the surrounding refractive index. When the refractive index changes, the resonant frequency will shift, resulting in a phase shift.

Simulation on the meta-unit is carried out with a commercial software, Lumerical FDTD Solutions (Lumerical, Vancouver, BC, Canada). The refractive index of the silicon is set as $n_{\mathrm{Si}}=3.45$. The LC is set as a diagonal dielectric material with $n_{\mathrm{O}}=1.60$ (diagonal elements $x x$ and $y y$ ) and $n_{\mathrm{e}}=1.90$ (diagonal element $z z$ ). The LC director distributions are set by an LC orientation module. An $x$-polarized plane wave with a spectrum range from 0.8 to $1.2 \mathrm{THz}$ is incident along $z$-axis. Periodic boundary conditions are set on both $x$ - and $y$-axes and a perfectly matched layer is set on $z$-axis. 

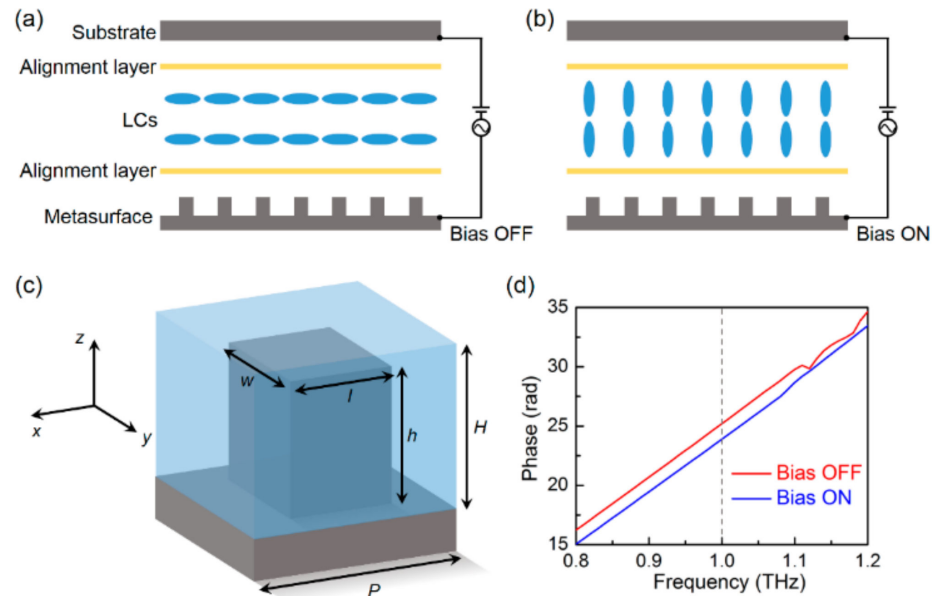

Figure 1. (a,b) Decomposed diagram of the liquid crystal integrated metalens at (a) bias OFF and (b) bias ON states, respectively. (c) Schematic of the meta-unit where the unit dimension is shown: periodicity, $P: 130 \mu \mathrm{m}$; height of the silicon pilar, $h: 235 \mu \mathrm{m}$; length of the pilar, $l: 120 \mu \mathrm{m}$; width of the pilar, w: $40 \mu \mathrm{m}$; height of the LC layer, $H: 250 \mu \mathrm{m}$. (d) Frequency-dependent phase shift when the $x$-polarized wave transmits through the meta-unit with the dimension shown in (c) at bias OFF (red line) and ON (blue line) states.

We fix $h=235 \mu \mathrm{m}, P=130 \mu \mathrm{m}, H=250 \mu \mathrm{m}$ and target frequency at $1.0 \mathrm{THz}$, and then vary $l$ and $w$ both from 10 to $120 \mu \mathrm{m}$ to achieve structural databases on the phase (Figure 2a,b) and the transmittance (Figure 2c,d), respectively, for $x$-polarized incidence. Observed from Figure $2 \mathrm{a}, \mathrm{b}$, the phase with a certain $l$ and $w$ value at bias OFF is larger than that at bias ON. As both $l$ and $w$ decrease from 120 to $10 \mu \mathrm{m}$, the phase difference become larger (from 0.1 to $1.5 \mathrm{rad}$ ). This is due to the different phase response to the electrically switched LC orientation with different $l$ and $w$. We can properly select the pillar geometries to simultaneously achieve the desired lens phase distributions with two different focal lengths for bias OFF and ON cases. The criterion for the transmittance of each pillar is set to be over 0.7 to optimize the lens efficiency.
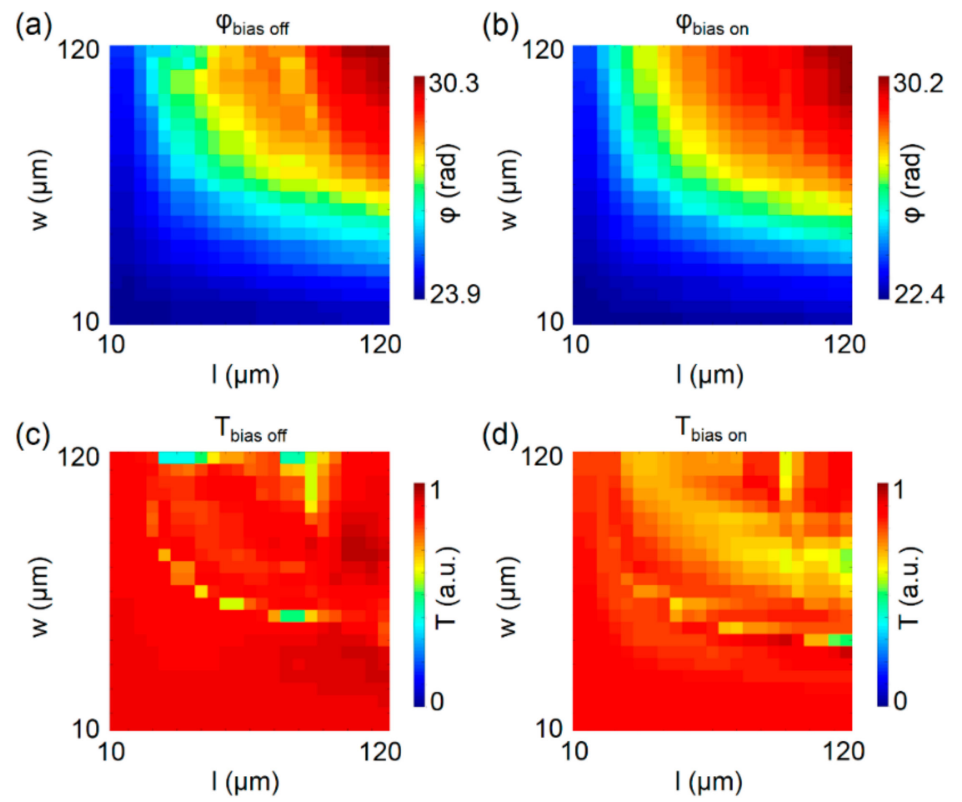

Figure 2. $(\mathbf{a}, \mathbf{b})$ Dependency of the phase shift on $l$ and $w$ when the $x$-polarized wave transmits through the meta-unit at (a) bias OFF and (b) bias ON states, respectively. (c,d) Dependency of the transmittance on $l$ and $w$ at (c) bias OFF and (d) bias ON states, respectively. 
To demonstrate the tunable focusing, two lenses with different focal lengths are designed. The phase profile of the lens is written as

$$
\varphi=-2 \pi\left(\sqrt{f_{i, j}^{2}+r^{2}}-f_{i, j}\right) / \lambda
$$

where $r$ is the radius position on the lens, $f$ is the focal length, the subscripts $i$ and $j$ indicate the different $f$ at bias OFF and ON states. Here, $f_{\mathrm{i}}$ and $f_{\mathrm{j}}$ are designed as $10.5 \mathrm{~mm}$ and $8.3 \mathrm{~mm}$, corresponding to the bias OFF and ON cases, respectively. The designed lens phase profiles with $f=10.5$ and $8.3 \mathrm{~mm}$ are presented in Figure 3a. They are both parabolic in shape with varied curvature. By rationally selecting the $l$ and $w$ parameters at different $r$ according to the database shown in Figure 2 and Equation (1), the parameters are decided and plotted in Figure $3 b$. The corresponding phases of different parameters are also plotted in Figure 3a. It can be seen that derived phases of these meta-units match well with the lens phase profiles at both bias OFF and ON states, indicating a satisfactory simulation parameter.
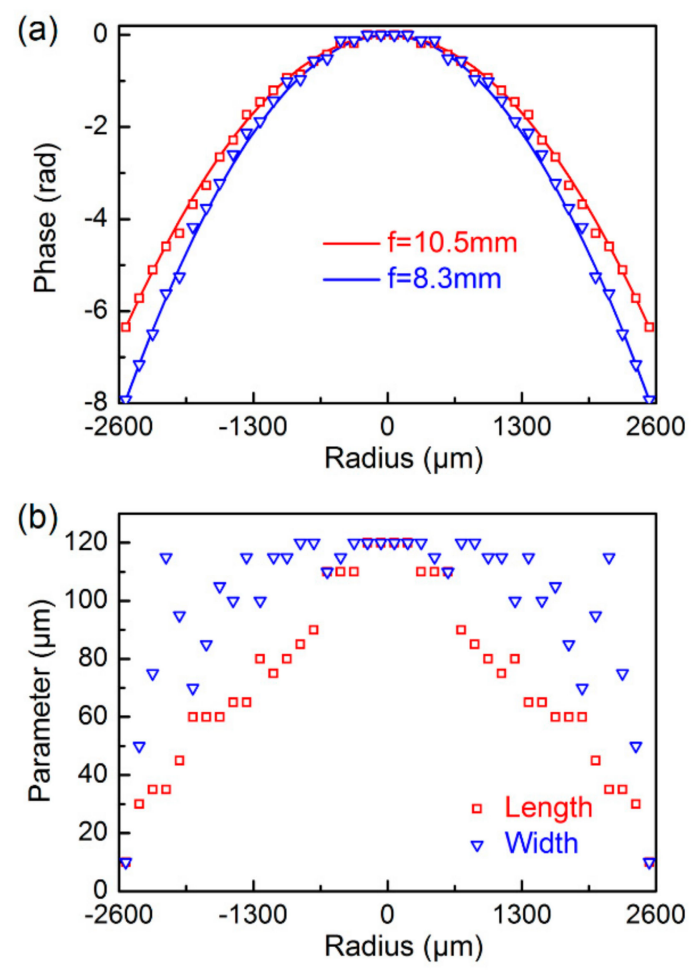

Figure 3. (a) Designed lens phase profiles with $f=8.3 \mathrm{~mm}$ (blue curve) and $10.5 \mathrm{~mm}$ (red curve) and phase shift of the meta-units with optimized dimensions along the radius at bias OFF (red dots) and bias ON (blue dots) states. (b) Optimized $l$ (red dots) and $w$ (blue dots) parameters of the meta-units along the radius.

\section{Results and Discussion}

We further characterize the focusing performance of the designed LC integrated metalens using the FDTD simulation. Due to the radial geometric symmetry of the lens, only a case along one radius is simulated. Figure $4 \mathrm{a}, \mathrm{b}$ are the simulated normalized $\mathrm{THz}$ fields in the $x z$-plane at $1.0 \mathrm{THz}$ at bias OFF and bias ON states, respectively, under the $x$-polarized wave incidence. The simulated $f_{\mathrm{i}}$ and $f_{\mathrm{j}}$ are $10.3 \mathrm{~mm}$ and $8.1 \mathrm{~mm}$, respectively, which are consistent with the design. We also characterize the transverse intensity distributions (Figure 4c) at the focal planes labeled by the white dashed lines in Figure 4a,b. The full widths at half maximum (FWHMs) of the transverse intensity at bias OFF and ON states are 514 and $428 \mu \mathrm{m}$, respectively, which are close to the ideal diffraction limit at $1.0 \mathrm{THz}$ $(\lambda / 2 \mathrm{NA})$. This indicates a satisfactory focusing effect at both the bias OFF and ON states. 
It is noticed that the real alignment may not be homogeneous like the simulated model. Since it is a proof of concept demonstration and has little influence on the focal tunable property of the lens, an ideal homogeneous LC orientation is considered at both bias OFF and $\mathrm{ON}$ states.
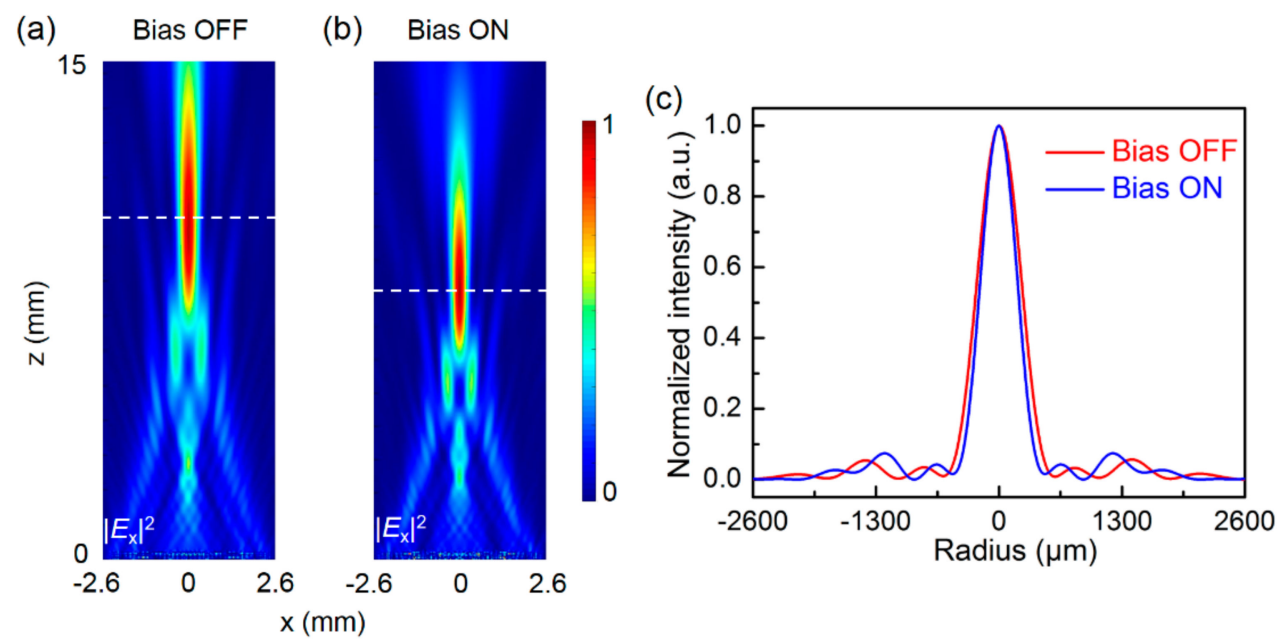

Figure 4. (a,b) Simulated THz fields in the $x z$-plane at $1.0 \mathrm{THz}$ at (a) bias OFF and (b) bias ON states, respectively. (c) Transverse intensity distributions at the focal planes labeled by the white dashed lines in (a) and (b) at bias OFF (red line) and bias ON (blue line) states.

In addition to the numerical simulations, the feasible fabrication process of the LC integrated metalens is also discussed and schematically illustrated in Figure 5. The dielectric metasurface can be fabricated via the standard photolithography process followed by a reactive ion etching on an intrinsic silicon wafer. Afterwards, both the metasurface and silicon substrate are ultrasonically cleaned and O-plasma treated. After that, they are spin-coated with a photoalignment layer. Then, an exposure of a linearly polarized blue LED is applied to introduce homogeneous alignment on both the metasurface and substrate. Then, the metasurface and silicon substrate are assembled with a Mylar spacer to form a cell. Finally, the large-birefringence LC with a birefringence of 0.3 at $1.0 \mathrm{THz}$ is infiltrated and self-assembled to the desired orientations. The experimental characterization of the fabricated device can be carried out with the photoconductive antenna or optical rectification-based $\mathrm{THz}$ generator and detector.

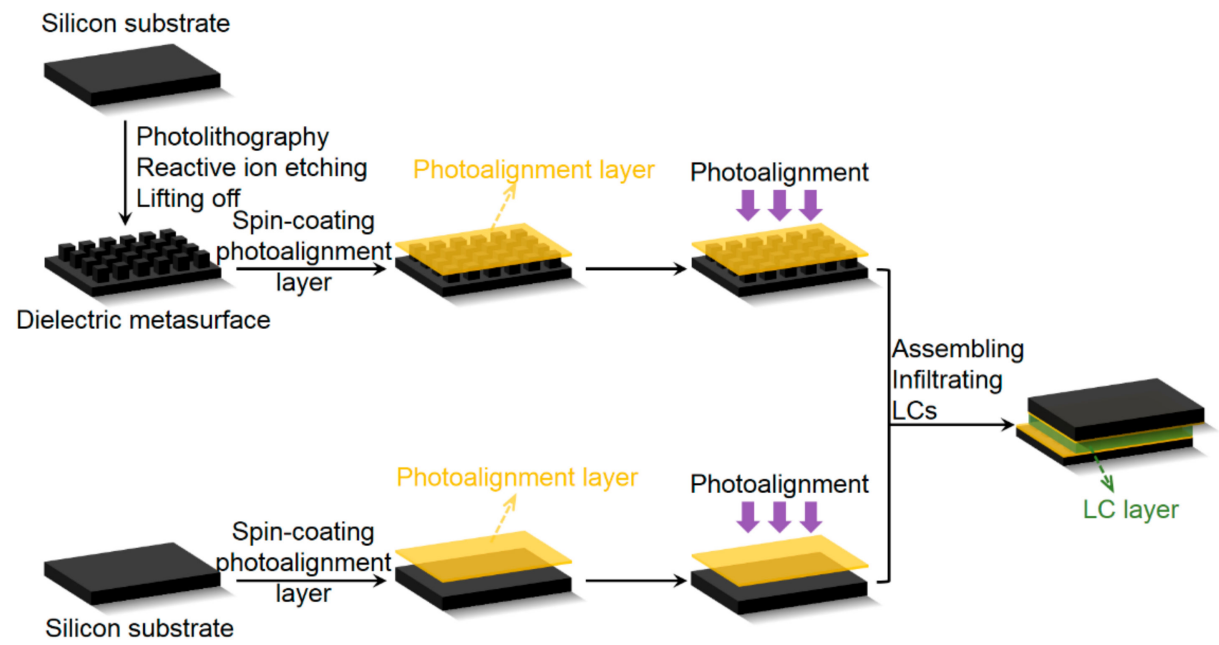

Figure 5. A feasible fabrication procedure of the LC integrated metalens. 


\section{Conclusions}

In summary, we propose an LC integrated metalens with electrically tunable focal length. The meta-unit of the metalens is composed of a silicon pillar and environmental LCs. When the LC is electrically switched under an applied bias, the phase of the transmitted $\mathrm{THz}$ wave will alter. By elaborately designing the geometric parameters of the meta-unit array, the tunable function of the wavefront modulations can be achieved. We numerically verified a tunable lens with its focal length changing from 10.3 to $8.1 \mathrm{~mm}$ from bias $\mathrm{OFF}$ to ON state, which reveals a good consistency with the design. Additionally, the fabrication process of the metalens is briefly discussed. Such a metalens has the great potential in advanced $\mathrm{THz}$ spectroscopy and imaging apparatuses, since the THz imagers are highly desired in biomedical and safety inspection applications. Furthermore, due to the broadband usability of LCs and metasurfaces, the design of this metalens can also be extended to other electromagnetic spectra. In the near-IR or visible bands, lenses with integrated tunable focal length are highly desired for applications such as full-color imaging and tomography. In the microwave band, active lenses can be used for the beam forming in radar applications.

Author Contributions: Conceptualization, Y.S. and Y.W.; methodology, Y.S. and Y.W.; software, Z.S.; validation, Y.S., Z.S. and W.H.; data curation, Y.W. and D.X.; writing-original draft preparation, Y.S. and Z.S.; writing-review and editing, Y.W. and D.X.; visualization, Y.S.; supervision, Z.S. and W.H.; funding acquisition, Y.S. All authors have read and agreed to the published version of the manuscript.

Funding: The authors gratefully acknowledge the support of the Research Foundation of Education Bureau of Guangdong Province, China (Grant No. 2020KTSCX287).

Data Availability Statement: The data presented in this study are contained within this article.

Acknowledgments: Not applicable.

Conflicts of Interest: The authors declare no conflict of interest.

\section{References}

1. Aieta, F.; Kats, M.A.; Genevet, P.; Capasso, F. Multiwavelength achromatic metasurfaces by dispersive phase compensation. Science 2015, 347, 1342-1345. [CrossRef] [PubMed]

2. Khorasaninejad, M.; Capasso, F. Metalenses: Versatile multifunctional photonic components. Science 2017, 358, eaam8100. [CrossRef] [PubMed]

3. Tseng, M.L.; Hsiao, H.H.; Chu, C.H.; Chen, M.K.; Sun, G.; Liu, A.Q.; Tsai, D.P. Metalenses: Advances and applications. Adv. Opt. Mater. 2018, 6, 1800554. [CrossRef]

4. Lalanne, P.; Chavel, P. Metalenses at visible wavelengths: Past, present, perspectives. Laser Photonics Rev. 2017, 11, 1600295. [CrossRef]

5. Khorasaninejad, M.; Chen, W.T.; Devlin, R.C.; Oh, J.; Zhu, A.Y.; Capasso, F. Metalenses at visible wavelengths: Diffraction-limited focusing and subwavelength resolution imaging. Science 2016, 352, 1190-1194. [CrossRef] [PubMed]

6. Yu, N.; Capasso, F. Flat optics with designer metasurfaces. Nat. Mater. 2014, 13, 139. [CrossRef]

7. Jia, D.; Tian, Y.; Ma, W.; Gong, X.; Yu, J.; Zhao, G.; Yu, X. Transmissive terahertz metalens with full phase control based on a dielectric metasurface. Opt. Lett. 2017, 42, 4494-4497. [CrossRef]

8. Wang, S.; Wang, X.; Kan, Q.; Ye, J.; Feng, S.; Sun, W.; Han, P.; Qu, S.; Zhang, Y. Spin-selected focusing and imaging based on metasurface lens. Opt. Express 2015, 23, 26434-26441. [CrossRef]

9. Wang, S.; Wu, P.C.; Su, V.-C.; Lai, Y.-C.; Chu, C.H.; Chen, J.-W.; Lu, S.-H.; Chen, J.; Xu, B.; Kuan, C.-H. Broadband achromatic optical metasurface devices. Nat. Commun. 2017, 8, 187. [CrossRef]

10. Wang, S.; Wu, P.C.; Su, V.-C.; Lai, Y.-C.; Chen, M.-K.; Kuo, H.Y.; Chen, B.H.; Chen, Y.H.; Huang, T.-T.; Wang, J.-H. A broadband achromatic metalens in the visible. Nat. Nanotechnol. 2018, 13, 227. [CrossRef]

11. Chen, W.T.; Zhu, A.Y.; Sanjeev, V.; Khorasaninejad, M.; Shi, Z.; Lee, E.; Capasso, F. A broadband achromatic metalens for focusing and imaging in the visible. Nat. Nanotechnol. 2018, 13, 220. [CrossRef]

12. Cheng, Q.; Ma, M.; Yu, D.; Shen, Z.; Xie, J.; Wang, J.; Xu, N.; Guo, H.; Hu, W.; Wang, S. Broadband achromatic metalens in terahertz regime. Sci. Bull. 2019, 64, 1525-1531. [CrossRef]

13. Nemati, A.; Wang, Q.; Hong, M.; Teng, J. Tunable and reconfigurable metasurfaces and metadevices. Opto-Electron. Adv. 2018, 1, 180009. [CrossRef] 
14. Gu, D.; Wen, B.; Mahajan, M.; Taber, D.; Winker, B.; Guthals, D.; Campbell, B.; Sox, D. High power liquid crystal spatial light modulators. In Advanced Wavefront Control: Methods, Devices, and Applications IV; International Society for Optics and Photonics: Bellingham, WA, USA, 2006; p. 630602.

15. Ge, S.J.; Chen, P.; Shen, Z.X.; Sun, W.F.; Wang, X.K.; Hu, W.; Zhang, Y.; Lu, Y.Q. Terahertz vortex beam generator based on a photopatterned large birefringence liquid crystal. Opt. Express 2017, 25, 12349-12356. [CrossRef]

16. Chen, P.; Ji, W.; Wei, B.Y.; Hu, W.; Chigrinov, V.; Lu, Y.Q. Generation of arbitrary vector beams with liquid crystal polarization converters and vector-photoaligned q-plates. Appl. Phys. Lett. 2015, 107, 241102. [CrossRef]

17. Wei, B.Y.; Chen, P.; Hu, W.; Ji, W.; Lu, Y.Q. Polarization-controllable Airy beams generated via a photoaligned director-variant liquid crystal mask. Sci. Rep. 2015, 5, 17484. [CrossRef]

18. Duan, W.; Chen, P.; Wei, B.Y.; Ge, S.J.; Liang, X.; Hu, W.; Lu, Y.Q. Fast-response and high-efficiency optical switch based on dual-frequency liquid crystal polarization grating. Opt. Mater. Express 2016, 6, 597-602. [CrossRef]

19. Liu, Y.; Chen, P.; Yuan, R.; Ma, C.Q.; Lu, Y.Q. Ferroelectric liquid crystal mediated fast switchable orbital angular momentum of light. Opt. Express 2019, 27, 36903. [CrossRef]

20. Kowerdziej, R.; Jaroszewicz, L. Tunable dual-band liquid crystal based near-infrared perfect metamaterial absorber with high-loss metal. Liq. Cryst. 2019, 46, 1568-1573. [CrossRef]

21. Chikhi, N.; Lisitskiy, M.; Papari, G.; Tkachenko, V.; Andreone, A. A hybrid tunable THz metadevice using a high birefringence liquid crystal. Sci. Rep. 2016, 6, 34536. [CrossRef]

22. Kowerdziej, R.; Wrobel, J.; Kula, P. Ultrafast electrical switching of nanostructured metadevice with dual-frequency liquid crystal. Sci. Rep. 2019, 9, 20367. [CrossRef]

23. Shen, Z.-X.; Zhou, S.-H.; Ge, S.-J.; Hu, W.; Lu, Y.-Q. Liquid crystal enabled dynamic cloaking of terahertz Fano resonators. Appl. Phys. Lett. 2019, 114, 041106. [CrossRef]

24. Shen, Z.; Zhou, S.; Ge, S.; Duan, W.; Chen, P.; Wang, L.; Hu, W.; Lu, Y. Liquid-crystal-integrated metadevice: Towards active multifunctional terahertz wave manipulations. Opt. Lett. 2018, 43, 4695-4698. [CrossRef]

25. Shen, Z.; Zhou, S.; Li, X.; Ge, S.; Chen, P.; Hu, W.; Lu, Y.-Q. Liquid crystal integrated metalens with tunable chromatic aberration. Adv. Photonics 2020, 2, 036002. [CrossRef]

26. Arbabi, A.; Horie, Y.; Ball, A.J.; Bagheri, M.; Faraon, A. Subwavelength-thick lenses with high numerical apertures and large efficiency based on high-contrast transmitarrays. Nat. Commun. 2015, 6, 7069. [CrossRef] [PubMed] 